\title{
Reutilización de aguas grises domésticas para el uso eficiente del recurso hídrico: aceptación social y análisis financiero. Un caso en Portugal
}

\section{Domestic greywater reuse for water use efficiency: social acceptance and financial analysis. A case in Portugal}

\author{
Julisse Andrea Meléndez-Pérez ${ }^{1 a}$, María Manuela C. Lemos-Lima ${ }^{1 b}$, Isabel C. Domínguez ${ }^{2 a}$, E. Ricardo \\ Oviedo-Ocaña ${ }^{2 b}$ \\ ${ }^{1}$ Departamento de Engenharia Civil, Escola de Engenharia, Universidade do Minho, Portugal. \\ Correo: ${ }^{1 a}$ j.andrea.melendez@ hotmail.com, ${ }^{1 b}$ mmlima@ civil.uminho.pt \\ ${ }^{2}$ Grupo de investigación en recursos hídricos y saneamiento ambiental (GPH), Facultad de Ingeniería \\ Fisicomecánicas, Universidad Industrial de Santander, Colombia. \\ Orcid: ${ }^{2 \mathrm{a}}$ 0000-0002-7677-2731, ${ }^{2 \mathrm{~b}} 0000-0002-8970-7322$ \\ Correos electrónicos: ${ }^{2 \mathrm{a}}$ isabeldr@uis.edu.co. ${ }^{2 \mathrm{~b}}$ eroviedo@uis.edu.co
}

Recibido: 5 abril, 2018. Aceptado: 4 julio, 2018. Versión final: 30 septiembre, 2018.

\begin{abstract}
Resumen
La reutilización de aguas grises (AG) contribuye a la preservación del agua y a disminuir la demanda de agua potable. En busca de potenciar esta alternativa en Portugal, se diseñó y evaluó financieramente un sistema hidrosanitario con reutilización de AG, en un edificio multifamiliar, considerando la aceptación de los usuarios y los sistemas de tratamiento del AG para cumplir con los estándares de calidad para reúso. Los potenciales usuarios aceptaron el reúso de AG provenientes de la ducha, lavamanos y bidé, en la descarga de inodoros y riego de jardines. El sistema propuesto incluye el tratamiento del AG con reactores biológicos de membrana (MBR). Se presentó un periodo de retorno de 24 años y un valor presente neto de 14.775,18 USD, con lo que se demuestra la viabilidad del sistema y se logra una reducción anual en el consumo de agua del $33 \%\left(\right.$ i. e. $3.351,92 \mathrm{~m}^{3}$ ) y se contribuye al uso eficiente del recurso en el ámbito urbano.
\end{abstract}

Palabras clave: aceptación social; aguas grises; factibilidad financiera; reúso.

\begin{abstract}
Greywater reuse (GWR) in the domestic sector is increasingly recognized as an alternative to face the growing water scarcity. GWR provides an alternative water source (AWS) for purposes that do not require potable water quality. Aiming at promoting AWS implementation in Portugal, a GWR hydro-sanitary system was designed and financially assessed for a multifamily building (72 flats). Social acceptancy of sanitary devices and treatment systems that fulfil quality standards for water reuse was addressed. Consultation with potential users allowed identifying GWR acceptance for water from shower, washbasin and bidet for end-uses such as toilet flushing and garden irrigation. The system included a commercial treatment consisting on Membrane Bioreactor, and had 24 years payback period, Net Present Value of $14.775,18$ USD, and annual potable water savings of $33 \%\left(3.351,92 \mathrm{~m}^{3}\right)$. Thus, the designed system was technical, social and financially feasible and can contribute to urban water use efficiency.
\end{abstract}

Keywords: social acceptance; greywater; financial feasibility; reuse.

ISSN impreso: 1657 - 4583, ISSN en línea: 2145 - 8456, CC BY-ND 4.0 (c) ()

Como citar: J. A .Meléndez, M. M. C. Lemos-Lima, I. Domínguez, R. Oviedo-Ocaña, "Reutilización de aguas grises domésticas para el uso eficiente del recurso hídrico: aceptación social y análisis financiero. Un caso en Portugal," Rev. UIS Ing., vol. 18, no. 1, pp. 223-236, 2019. doi: 10.18273/revuin.v18n1-2019020 


\section{Introducción}

El mundo se enfrenta a un déficit de agua que podría agravarse por el escenario de cambio climático y el manejo actual del recurso hídrico [1]. Actualmente, dos tercios de la población viven en regiones donde sufren escasez de agua al menos una vez al año. Asimismo, tan solo el $20 \%$ del agua residual (AR) se trata antes de su vertimiento a cuerpos de agua [2], lo que degrada la calidad de las fuentes hídricas y limita la oferta disponible. Esta situación plantea nuevos retos en la gestión del recurso hídrico y el uso eficiente del agua. Por ejemplo, la Unión Europea (UE) ha establecido ser más eficiente en el manejo de los recursos hídricos, de forma que se requiere que para el 2020 la extracción de agua se mantenga por debajo del $20 \%$ de los recursos hídricos renovables disponibles [1].

En Portugal, el consumo de agua en el sector urbano $(8 \%)$ es menor que en el agrícola $(87 \%)$ y relativamente similar al industrial (5\%) [3]; sin embargo, los costos asociados al ciclo urbano del agua, desde su captación, potabilización, distribución, así como recolección y tratamiento de aguas residuales, representan el $46 \%$ de los costos totales de suministro de agua para todos los sectores ( $i$. e e. la agricultura representa el $28 \%$ y la industria el $26 \%$ ) [3]. Asimismo, el consumo de agua en el ámbito doméstico representa la mayor proporción del consumo urbano (i. e. cerca del $64 \%$ ) [4]. Por tanto, la implementación de medidas de uso eficiente de agua en el sector urbano, en particular en el ámbito doméstico, podría tener un efecto en la reducción de costos del ciclo urbano del agua.

La reutilización de AR se constituye en una estrategia sustentable de gestión de los recursos hídricos, motivada esencialmente por la escasez de agua [5]. WWAP [2] reconocen el reúso de AR como una de las fases clave para el manejo de AR, que se constituye en un pilar fundamental de la economía circular.

El reúso de $\mathrm{AR}$ se ha empleado tradicionalmente en sistemas centralizados con diversas aplicaciones (e. $g$. piscicultura y agricultura) [5]; [6], lo que ha contribuido a disminuir el estrés hídrico resultante de la alta demanda para estos usos. Sin embargo, varias experiencias han sido implementadas en esquemas descentralizados principalmente en ciudades superpobladas como Japón y Singapur [5]. En estos esquemas, el agua contaminada es captada, tratada y distribuida en el mismo predio para usos no potables (e. g. lavados de carros y pavimentos, descarga de inodoros, riega de jardín), lo que reduce el uso de agua potable en actividades en las que no se requiere agua de alta calidad, así como ayuda a disminuir el volumen de AR producidas [7].
De las AR domésticas, las que presentan mayor potencial de reutilización son las aguas grises $(\mathrm{AG})$, debido a su menor contaminación -si se las compara con las aguas negras-, mayor facilidad de tratamiento y menor riesgo asociado a su reutilización [8]. Las AG provenientes del lavamanos, ducha y bidé han sido reusadas para descarga de inodoros, riego de jardín [9], [10], [11], limpieza del hogar, lavandería y lavado de vehículos [8], [12]. Sin embargo, en estos estudios no se ha consultado la preferencia de los usuarios por los aparatos sanitarios involucrados en el sistema (i. e. para captación y reúso).

La implementación de sistemas descentralizados ha sido financieramente factible en residencias estudiantiles, hoteles, unidades residenciales y viviendas unifamiliares [7], [9], [12], [13], [14], [15], [16]; no obstante, se observan mejores indicadores financieros cuando se reutilizan mayores volúmenes de agua [10].

En Portugal, de acuerdo con diferentes referencias [17], [18], cerca del $50 \%$ de los usos del agua en las residencias no requiere agua estrictamente potable, por lo que se podría emplear agua alternativa de menor calidad en esos usos. Sin embargo, Portugal no cuenta con una normativa completa sobre reutilización de AG en el ámbito doméstico y restringe el reúso de las $\mathrm{AR}$ exclusivamente a lavado de pavimentos, riego, combate de incendios y fines industriales no alimentarios, lo que excluye usos domésticos, como la descarga de inodoros.

En este artículo se analiza la factibilidad financiera de la reutilización de AG en el sector doméstico en Portugal. En el conocimiento de los autores, hay información limitada sobre este tipo de estudios en el contexto de estudio. El artículo incluye la propuesta de una alternativa hidrosanitaria para la recolección de AG en edificios multifamiliares, su tratamiento y distribución para reúso. La alternativa fue construida a partir de la consulta de potenciales usuarios del sistema. Una vez diseñada la alternativa, se determinaron sus costos y analizaron indicadores financieros de su implementación. Este tipo de estudios provee información sobre la planeación y factibilidad financiera que deben realizarse para la implementación de sistemas alternativos de suministro de agua con visión de sostenibilidad.

\section{Metodología}

\subsection{Descripción del caso de estudio}

El estudio se realizó en un proyecto de edificio residencial con 72 apartamentos por construir en la ciudad de Matosinhos, situada en el distrito de Porto; 
(a)

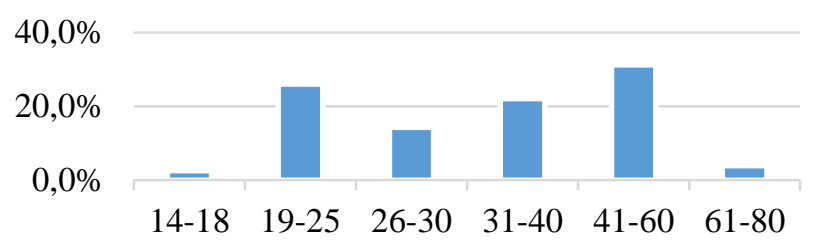

(b)

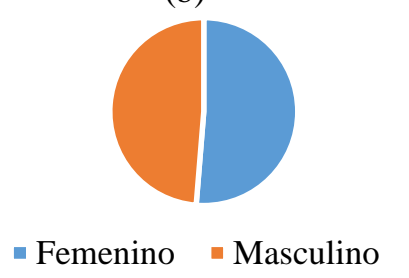

(c)

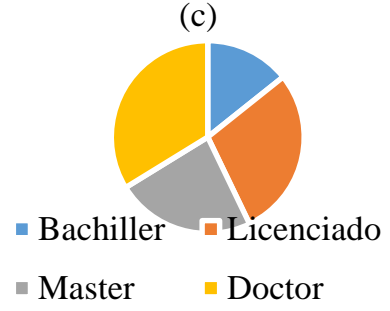

Figura 1. Información personal de la muestra, (a) edad, (b) sexo y (c) formación académica. Fuente: elaboración propia.

representa una vivienda de nivel socioeconómico medio. El edificio está compuesto por dos sótanos de parqueaderos (piso -2 y -1), tres espacios comerciales, el lobby del edificio y un jardín (piso 0), y apartamentos residenciales entre el piso 1 y el 9 . Cada piso cuenta con 8 apartamentos (i. e. dos T1: 1 habitación, dos T2: 2 habitaciones, tres $\mathrm{T} 2+1: 2$ habitaciones + un espacio menor a una habitación y un T3: 3 habitaciones). La zona de recreo se encuentra en el piso 10 y consta de piscina, solárium y vestuarios.

\subsection{Encuesta de reutilización de AG}

Se aplicó una encuesta para determinar la aceptación social del reúso de $\mathrm{AG}$, el consumo medio de agua potable por habitante y la preferencia de opciones oferta y demanda para el reúso de AG. El instrumento fue enviado a la comunidad de la Universidade do Minho, a través del correo electrónico institucional, debido a la dificultad de realizar las encuestas personalmente en viviendas con características socioeconómicas similares a las del proyecto. Se recibió un total de 78 respuestas. En la encuesta se incluyeron preguntas de información personal, para verificar la heterogeneidad de la muestra. De acuerdo con la figura 1, la muestra es en su mayoría de edad adulta, y la mayor proporción se concentra entre una edad de 31 y 60 años. Además, está conformada prácticamente en la misma proporción tanto de hombres como de mujeres y cuenta con individuos de todas las formaciones académicas, lo que demuestra su heterogeneidad.

\subsubsection{Aceptación social del reúso de AG}

Se determinó que el $80 \%$ de los encuestados tienen disposición de reusar las AG, a fin de privilegiar la protección del medioambiente sobre el posible ahorro económico del sistema. Este porcentaje es similar a los resultados obtenidos en investigaciones en Colombia (86 \%), Omán (84 \%) y Siria (83\%) [7], [13], [19]. Del $20 \%$ de encuestados que se oponen a reutilizar las AG, cerca del $87 \%$ argumentaron aspectos de higiene. De acuerdo con Gross et al. [20], la sociedad tiene una barrera psicológica que genera una sensación de repulsión hacia el uso de agua reciclada, debido a una percepción de que el agua está sucia o que puede presentar alguna infección después del uso, a pesar de que no exista un riesgo real, en el caso de tratar eficazmente el agua.

\subsubsection{Consumo medio por habitante}

La dotación obtenida fue de 102,77 \pm 57,18 L/hab/día, valor cercano al reportado en diferentes estudios en el contexto de Portugal (tabla 1), por lo que se optó por trabajar con este valor.

Tabla 1. Comparación de la dotación obtenida y otros estudios en Portugal

\begin{tabular}{ll}
\hline Resultados de capitaciones & L/hab.día \\
\hline Encuesta & 102,77 \\
INSAAR (2011) & 169 \\
Melo baptista et al. (2001) & 78 \\
Friedler y Hadari (2006) & $100-180$ \\
Matos (2009) & 114,7 \\
\hline
\end{tabular}

Fuente: elaboración propia. 


\subsubsection{Opciones de reutilización de AG}

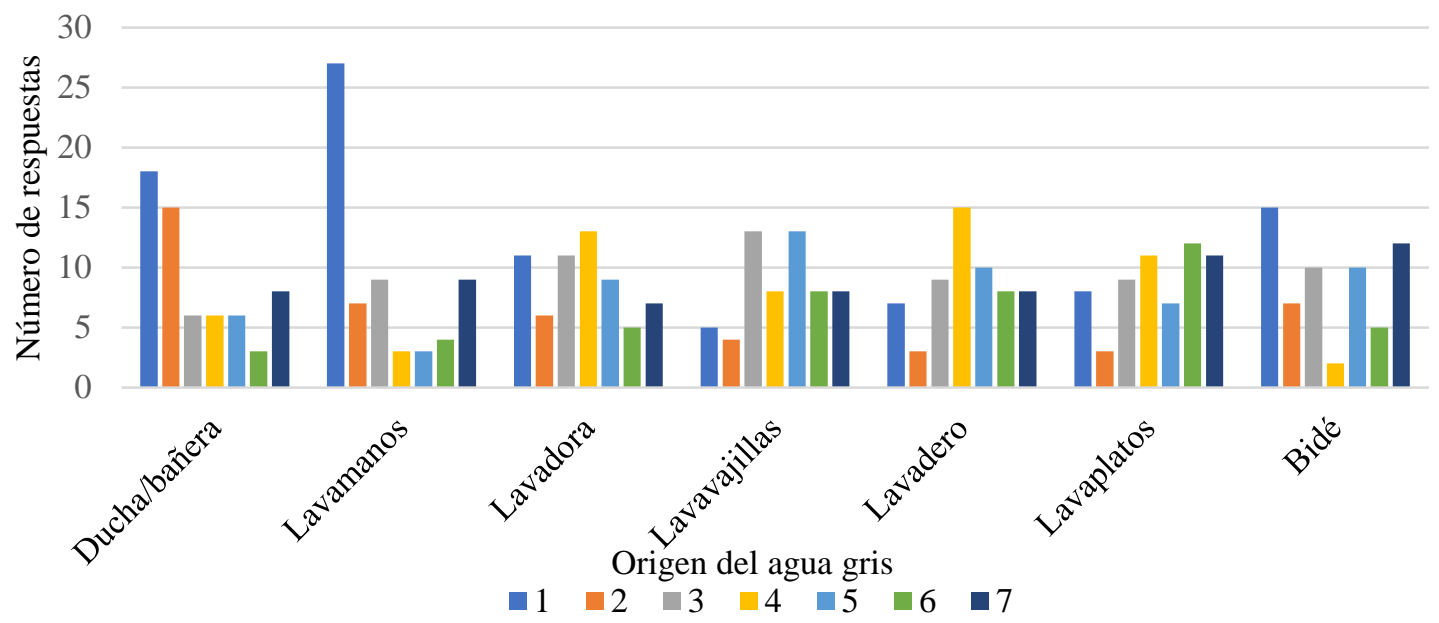

Figura 2. Preferencia del origen del agua gris para reutilización según la encuesta realizada. Fuente: elaboración propia.

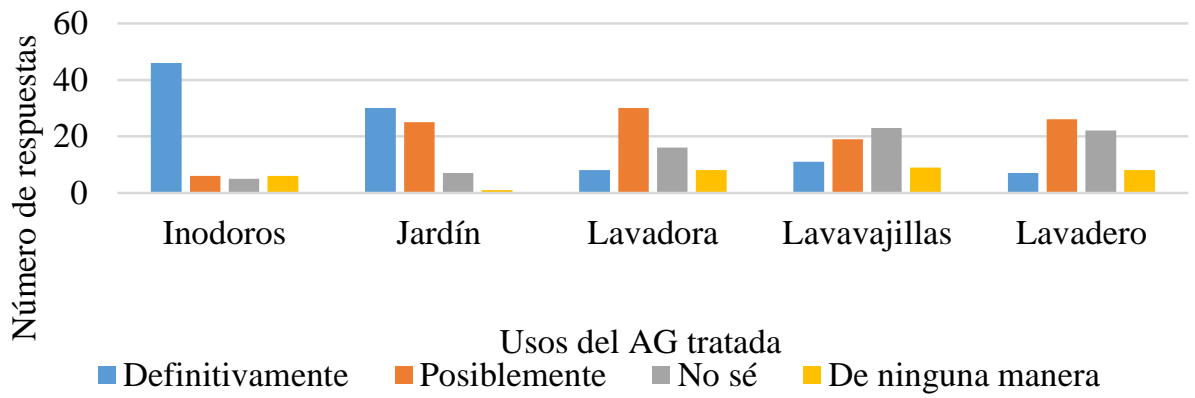

Figura 3. Usos de preferencia para el AG tratada. Fuente: elaboración propia.

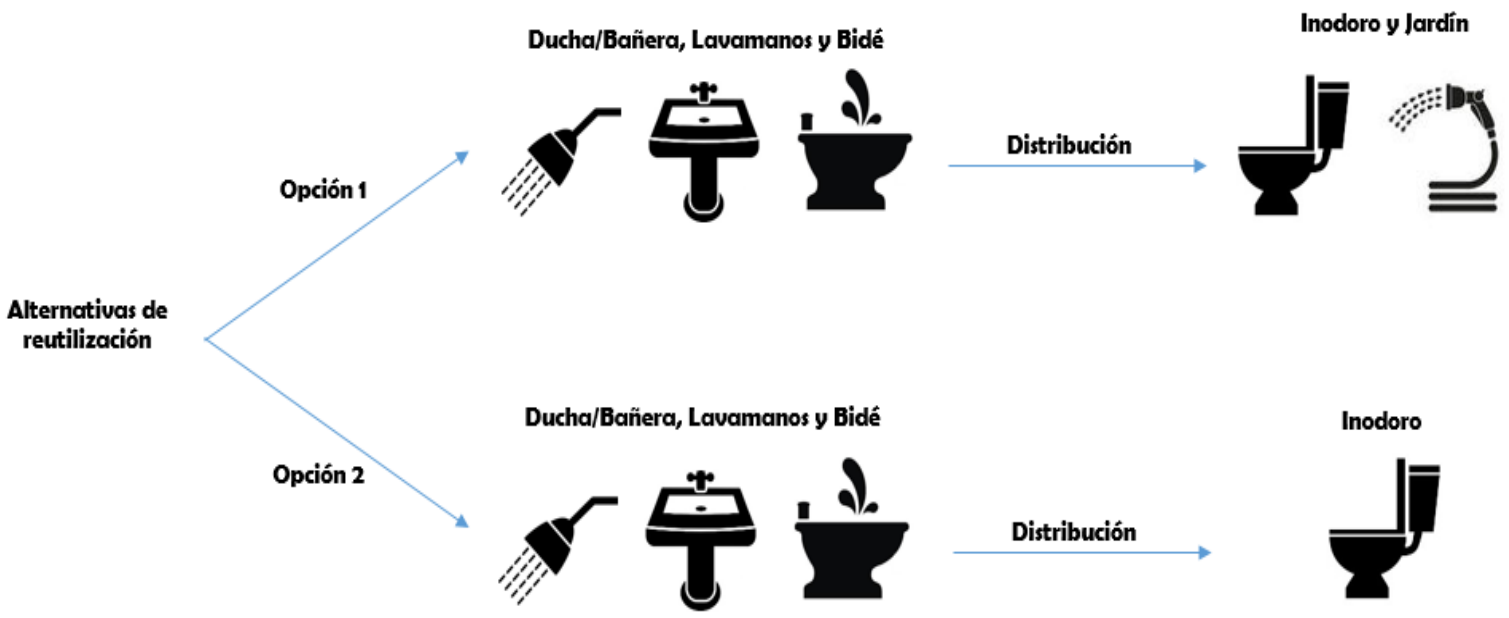

Figura 4. Opciones de reutilización propuestas. Fuente: elaboración propia. 
Los encuestados calificaron la preferencia del origen del AG para el tratamiento y posterior reúso (i. e. de 1 a 7 , donde 1 es la opción de mayor preferencia) (figura 2). Se encontró una mayor aceptación por los aparatos del baño en los que se incluyen, en orden descendente: lavamanos, bañera/ducha y bidé. Estos aparatos fueron seleccionados debido a que presentan características fisicoquímicas favorables para su reutilización, por estar categorizados como AG claras [8], [21].

De otro lado, se determinó que los usos de preferencia para el reúso de AG tratada corresponden a la descarga de inodoros y el riego del jardín (figura 3), lo que está en concordancia con los esquemas de tratamiento en diferentes partes del mundo revisados por Pidou et al. [22].

\subsection{Sistema de reutilización de AG}

\subsubsection{Estimación de la oferta y demanda del sistema}

El cálculo de la demanda y oferta se realizó a partir de la distribución del consumo de agua a ámbito doméstico, la dotación neta por habitante y el número de residentes según la tipología de los apartamentos (i. e. 2-3 habitantes para T1, 4 habitantes para $\mathrm{T} 2$ y $\mathrm{T} 2+1$ y 5 habitantes para T3) para cada una de las opciones de reutilización propuestas.

El consumo de cada aparato sanitario involucrado en las opciones de reutilización se calculó a partir de referencias bibliográficas, y se obtuvieron los siguientes porcentajes del consumo total: 1) ducha/bañera: $33 \%$ [8], 2) lavamanos: $9 \%$ [8], 3) inodoro: $31 \%$ [23]. Asimismo, se tuvieron en cuenta las siguientes dotaciones: 1) bidé: 2,5 L/hab/día [21], [24], 2) riego: $0,2 \mathrm{~m}^{3} / \mathrm{m}^{2} / \mathrm{mes}$ en los 5 meses de menor precipitación (mayo a septiembre) [25].

Se determinó un número de 270 habitantes en la unidad multifamiliar y un área de jardines de $26,8 \mathrm{~m}^{2}$ en el piso 0 . No se consideró efectuar riego en el área de jardines ubicada en el piso 10, debido a la proximidad de las áreas verdes con actividades de recreación de contacto directo, como la piscina.

La oferta del sistema está representada como el volumen mensual total producido por los aparatos sanitarios productores de AG presentes en las opciones de reutilización. Del mismo modo, la demanda fue calculada con el volumen mensual total requerido por los aparatos sanitarios que cuentan con una mayor aceptación para el uso de las AG tratadas, teniendo en cuenta que estos usos no requieren agua estrictamente potable (tabla 2 ).
Tabla 2. Oferta y demanda del sistema de reutilización de aguas grises

\begin{tabular}{lcc}
\hline Aparato sanitario & $\begin{array}{c}\text { Capitación } \\
(\text { L/hab.día })\end{array}$ & $\begin{array}{c}\text { Consumo } \\
\left(\mathrm{m}^{3} / \text { día }\right)\end{array}$ \\
\hline Ducha/bañera & & \\
$(33 \%)$ & 33,91 & 9,16 \\
Lavamanos $(9 \%)$ & 9,25 & 2,50 \\
Bidé & 2,5 & 0,68 \\
Inodoro $(31 \%)$ & 31,86 & 8,60 \\
Jardín $\left(\mathrm{m}^{3} / \mathrm{m}^{2}\right.$. mes) & 0,2 & 1,45 \\
\hline Opción 1 & Oferta & 12,33 \\
& Demanda & 10,05 \\
\hline Opción 2 & Oferta & 12,33 \\
& Demanda & 8,60 \\
\hline
\end{tabular}

Fuente: elaboración propia.

\subsubsection{Selección del sistema de tratamiento}

Como sistema de tratamiento de las AG, se optó por implantar un sistema comercial con una capacidad de $10000 \mathrm{~L} /$ día, que tiene consumo eléctrico menor de 1.7 $\mathrm{kWh} / \mathrm{m}^{3}$. El sistema de tratamiento está compuesto por tratamiento preliminar (desbaste), un reactor aerobio, seguido por ultrafiltración con membranas y desinfección con hipoclorito de sodio, para un posterior almacenamiento.

Acorde con la tabla 3, el sistema propuesto por el proveedor cumple con los valores máximos admisibles para E. coli (200 UFC/100 mL), parásitos entéricos (1 huevo/10 L), SST (10 mg/L) y turbidez (2 UNT) definidos por la Asociación Nacional para la Calidad en las Instalaciones Prediales (ANQIP) como los requisitos destinados del agua para riego y descarga de inodoros. Sin embargo, no existe una legislación completa en Portugal con los diferentes parámetros para realizar una comparación más exacta.

Tabla 3. Características a cumplir por el efluente acorde con ANQIP

\begin{tabular}{lll}
\hline Parámetro & Unidad & Valor \\
\hline $\mathrm{DBO}_{5}$ & $\mathrm{mg} / \mathrm{L}$ & $<15$ \\
$\mathrm{DQO}$ & $\mathrm{mg} / \mathrm{L}$ & $<50$ \\
$\mathrm{SST}$ & $\mathrm{mg} / \mathrm{L}$ & $<2$ \\
Turbidez & $\mathrm{UNT}$ & $<1$ \\
E. coli & UFC/100 mL & 0 \\
Huevos de Nematodos & huevo/10 L & $<1$ \\
\hline
\end{tabular}

Fuente: Memoria técnica GREM 10000.

Así mismo, cumple con los valores de referencia propuestos por Oh et al. [26], a partir de una revisión de 
normas de calidad para reúso de aguas grises en diferentes contextos, y presenta valores de referencia para reúso en fines no potables, como SST $<20 \mathrm{mg} / \mathrm{L}$, DBO5 $<20 \mathrm{mg} / \mathrm{L}$, pH entre 6 y 9 , y turbiedad $<5$ UNT y $<10 \mathrm{UFC} / 100 \mathrm{~mL}$ de Escherichia coli (E. coli). [24].

\subsubsection{Ahorro del sistema por reducción en el pago de tarifas de servicios públicos}

El ahorro de agua se calculó con el menor valor entre la oferta y la demanda, tomando la tarifa de venta de agua según el marco tarifario para 2017 del prestador del servicio de la localidad objeto de estudio (tabla 4).

Tabla 4. Tarifa diferenciada de venta de agua

\begin{tabular}{ll}
\hline Escalones $\left(\mathrm{m}^{3} / \mathrm{mes}\right)$ & Precio $\left(\mathrm{USD} / \mathrm{m}^{3}\right)$ \\
\hline $1^{\circ}$ Escalón- 0 a $5 \mathrm{~m}^{3}$ & 0,6674 \\
$2^{\circ}$ Escalón- 6 a $15 \mathrm{~m}^{3}$ & 1,0740 \\
$3^{\circ}$ Escalón- 16 a $25 \mathrm{~m}^{3}$ & 1,9623 \\
$4^{\circ}$ Escalón- superior a $25 \mathrm{~m}^{3}$ & 2,9400 \\
\hline
\end{tabular}

Nota: Valores sujetos a IVA tasa reducida- $6 \%$ Fuente: INDAQUA (2017)

Acorde con la tabla 2, la oferta supera la demanda en el caso de las dos opciones de reúso propuestas. La demanda total de la Opción 1 (10,05 m³/día) es mayor a la capacidad del sistema de tratamiento $\left(10 \mathrm{~m}^{3} / \mathrm{dí}\right)$, de modo que el ahorro de agua potable diario será igual a la capacidad del sistema de tratamiento. Por tanto, se tendrá un ahorro promedio de $300 \mathrm{~m}^{3} /$ mes en los 5 meses de menor precipitación; y de $258 \mathrm{~m}^{3} / \mathrm{mes}$, en el resto del año para un total de $3.351,92 \mathrm{~m}^{3} /$ año. En la opción 2 , el ahorro de agua potable es de $8,6 \mathrm{~m}^{3} /$ día para un ahorro anual de $3.139 \mathrm{~m}^{3}$.

Debido a que el cobro del agua se hace por escalones de consumo, el precio del agua se calculó de acuerdo con las (ecuaciones 1 a 4 ):

$$
\begin{array}{rl}
\text { Precio }_{m}=\text { Vol }_{m} & * 0.6674 ; 0<\text { Vol }_{m} \leq 5 \\
\text { Precio }_{m}=5 * & 0.6674+\left(\text { Vol }_{m}-5\right) \\
& * 1.0740 ; 6<\text { Vol }_{m} \leq 15 \\
\text { Precio }_{m}=5 * 0.6674+10 * 1.0740 \\
+
\end{array}
$$

Donde,

Precio $_{m}$, precio mensual (USD);

$\operatorname{Vol}_{m}$, consumo mensual $\left(\mathrm{m}^{3}\right)$.

El consumo en descarga de inodoros se calculó de acuerdo con la dotación per cápita y la población definida por tipología $\left(i\right.$. e. T1=2,39 $\mathrm{m}^{3} / \mathrm{mes} ; \mathrm{T} 2=3,82 \mathrm{~m}^{3} / \mathrm{mes}$; $\left.\mathrm{T} 3=4,78 \mathrm{~m}^{3} / \mathrm{mes}\right)$. En la encuesta se reportaron diferentes consumos totales para una misma tipología de apartamentos; por tanto, se definieron porcentajes de apartamentos por tipología para cada consumo y con las ecuaciones 1 a 4 se determinó el ahorro económico por costos de abastecimiento para descarga de inodoros.

El ahorro de agua potable por reúso en el jardín fue de 1,4 $\mathrm{m}^{3} /$ día, debido a la capacidad del sistema de tratamiento; el ahorro económico se calculó con el $4^{\circ}$ escalón suponiendo que el edificio presenta un alto consumo para zonas comunes, de acuerdo con sus instalaciones, para un total de 123,48 USD /mes en los meses de menor precipitación (en los meses de precipitación, no hay riego de jardín).

\begin{tabular}{|c|c|c|}
\hline Ahorro & Opción 1 & Opción 2 \\
\hline Volumen (m³/año) & $3.351,92$ & 3.139 \\
\hline $\begin{array}{l}\text { Costo de abastecimiento } \\
\text { (USD/año) }\end{array}$ & $4.069,73$ & $3.452,38$ \\
\hline $\begin{array}{l}\text { Costo } \\
\text { (USD/año) }\end{array}$ & 736,65 & 689,86 \\
\hline $\begin{array}{l}\text { Ahorro total con IVA incluido } \\
(€ / \text { año })\end{array}$ & $5.094,76$ & $4.390,77$ \\
\hline
\end{tabular}

La tasa de utilización para saneamiento del marco tarifario 2017 del prestador de servicio correspondiente fue de $0,22 \mathrm{USD} / \mathrm{m}^{3}$ (valores sujetos a tasa reducida- $6 \%$ ) de agua que entra al sistema de abastecimiento, con el cual se obtuvo el ahorro económico por costos de saneamiento para cada opción de reutilización como se muestra en la tabla 5 .

Tabla 5. Ahorro anual de agua potable

Fuente: elaboración propia.

\subsubsection{Determinación del consumo energético}

El consumo energético del sistema de reutilización de AG se calculó con la energía requerida por los sistemas de bombeo (e. $g$. requerida para garantizar la presión de $15 \mathrm{~m}$, en todos los dispositivos de utilización) y el sistema de tratamiento. 
Tabla 6. Consumo y costo de cada alternativa de bombeo

\begin{tabular}{|c|c|c|c|c|c|c|c|}
\hline \multirow[t]{3}{*}{ Alt } & \multirow{3}{*}{$\begin{array}{l}\text { Sistema de } \\
\text { bombeo }\end{array}$} & \multirow{2}{*}{\multicolumn{2}{|c|}{$\begin{array}{l}\text { Consumo según la } \\
\text { temporada }(\mathrm{kWh} / \mathrm{año})\end{array}$}} & \multirow{2}{*}{\multicolumn{2}{|c|}{ Opción 1}} & \multirow{2}{*}{\multicolumn{2}{|c|}{ Opción 2}} \\
\hline & & & & & & & \\
\hline & & Con riego & Sin riego & $\begin{array}{l}\text { Consumo } \\
(\mathrm{kWh} / \mathrm{año})\end{array}$ & $\begin{array}{c}\text { Costo } \\
\text { (USD/kWh) }\end{array}$ & $\begin{array}{l}\text { Consumo } \\
(\mathrm{kWh} / \mathrm{año})\end{array}$ & $\begin{array}{c}\text { Costo } \\
\text { (USD/kWh) }\end{array}$ \\
\hline 1 & $\begin{array}{l}\text { Hydro Multi- E3 } \\
\text { CRE 5-04 }\end{array}$ & $1.303,00$ & 1.126 .00 & $1.199,75$ & 220,49 & $1.126,00$ & 206,93 \\
\hline \multirow[t]{3}{*}{2} & $\begin{array}{l}\text { Hydro Multi- E2 } \\
\text { CRE 10-01 }\end{array}$ & 516,00 & 448.00 & 476,33 & 109,40 & 448,00 & 102,89 \\
\hline & $\begin{array}{l}\text { Hydro Solo E } \\
\text { CRE 1-9 HQQE }\end{array}$ & $1.274,00$ & 1.188 .00 & $1.223,83$ & 130,85 & $1.188,00$ & 127,01 \\
\hline & Total Alt. 2 & & & $1.700,17$ & 240,24 & 1636,00 & 229,90 \\
\hline
\end{tabular}

Fuente: elaboración propia.

Portugal cuenta con tarifas simples y "bihorarias" (i.e. se dividen las 24 horas del día en dos periodos, y los precios son diferentes en cada uno de ellos). El precio del consumo varía según el momento del día en el que fue hecho dicho consumo. Estos periodos se clasifican en: 1) horas de vacío: el precio de la energía es más bajo y es para consumos de energía entre las 22:00 y las 08:00 ( $i$. e. costo de 0,1069 USD kWh); 2) horas fuera de vacío: el precio de la energía es más alto y es para consumos de energía entre las 08:00 y las 22:00 (i. e. costo de 0,2297 USD/kWh). El costo de la tarifa simple es de 0,1838 $\mathrm{USD} / \mathrm{kWh}$.

Para la determinación del consumo energético por bombeo, se tuvieron en cuenta dos alternativas de diseño: 1) Alternativa 1: Un sistema de presurización desde el tanque del sistema de tratamiento hacia los dispositivos de utilización que funcione continuamente; 2) Alternativa 2: Un sistema de bombeo, desde el tanque del sistema de tratamiento, hacia un tanque de agua no potable ubicado en el piso 10, que funcione durante 10 horas al día (i. e. entre las 22:00 y las 8:00 horas), y un sistema de presurización desde el tanque hacia los dispositivos de utilización, para garantizar una presión superior a $15 \mathrm{~m}$, que funcione continuamente. Para cada alternativa, se determinaron el caudal y la altura manométrica de la bomba, con un dimensionamiento preliminar del sistema hidráulico.

Una vez estimados los consumos de energía, se determinaron los costos por tarifa de energía, considerando que la Alternativa 1 trabajará con tarifa simple, y que la Alternativa 2 trabajará con tarifa "bihoraria" (10 horas en el bombeo durante las horas de vacío), se estimaron los costos de cada alternativa (tabla 6). Se observa que, en ambas opciones, la Alternativa 1 presenta un menor consumo y un menor costo.

De otro lado, teniendo en cuenta que el consumo energético del sistema de tratamiento fue de $1,7 \mathrm{kWh} / \mathrm{m}^{3}$, se estimó el consumo total de energía para cada opción, de lo que se obtuvo como resultado un mayor consumo en la opción 1 (tabla 7).

Tabla 7. Consumo energético del sistema de tratamiento

\begin{tabular}{|c|c|c|c|c|}
\hline \multirow[t]{2}{*}{$\begin{array}{l}\text { Opción de } \\
\text { reutilización }\end{array}$} & $\begin{array}{l}\text { Consumo } \\
\text { temporada } \\
(\mathrm{kWh} / \mathrm{año})\end{array}$ & según la & $\begin{array}{l}\text { Consumo } \\
(\mathrm{kWh} / \mathrm{año} \text { ) }\end{array}$ & \multirow[t]{2}{*}{$\begin{array}{l}\text { Costo anual } \\
\text { (USD/kWh } \\
\text { ) }\end{array}$} \\
\hline & $\begin{array}{l}\text { Con } \\
\text { riego }\end{array}$ & $\begin{array}{l}\text { Sin } \\
\text { riego }\end{array}$ & & \\
\hline Opción 1 & $6.205,00$ & $5.336,30$ & $5.698,26$ & $1.047,21$ \\
\hline Opción 2 & N/A & $5.336,30$ & $5.336,30$ & 980,69 \\
\hline
\end{tabular}

Fuente: elaboración propia.

\subsubsection{Selección de la opción de reutilización}

Para seleccionar la opción de reutilización de AG más factible, se realizó un análisis preliminar de costos teniendo en cuenta el ahorro por reducción del pago de tarifas de abastecimiento y saneamiento y los costos de energéticos, sin tener en cuenta los costos constructivos (tabla 8).

Tabla 8. Evaluación financiera preliminar de las opciones en el primer año

\begin{tabular}{lccc}
\hline \multicolumn{2}{c}{ Ahorro } & Opción & Opción \\
& 1 & 2 \\
\hline Agua potable y & saneamiento & & \\
(USD/año) & & $5.094,76$ & $4.390,77$ \\
Consumo eléctrico (USD/año) & $1.343,83$ & $1.258,95$ \\
Ahorro total (USD/año) & $3.750,93$ & $3.131,82$ \\
\hline
\end{tabular}

Nota: No se incluyen para esta selección otras variables más detalladas. Los valores incluyen el Impuesto Sobre Valor Añadido (IVA).

Fuente: elaboración propia.

Por tanto, con este análisis preliminar, se seleccionó la Opción de reutilización 1, ya que cuenta con un ahorro 16,5\% mayor comparado con la Opción 2. 


\subsubsection{Diseño del sistema hidrosanitario con reutilización de AG}

El diseño del sistema hidrosanitario se realizó teniendo en cuenta los trazados existentes en los diseños originales y la normatividad para el diseño de sistemas hidrosanitarios de Portugal, en procura del mayor ahorro de materiales adicionales.

En el diseño original, las AR provenientes de lavamanos, duchas, bañeras y bidés en el sistema original se integran en un único bajante, separadas de las aguas negras del inodoro, hecho que facilitó el dimensionamiento del sistema de drenaje de AG hacia el sistema de tratamiento ubicado en el piso -1. A partir del tanque del sistema de tratamiento, y con ayuda del sistema de aumento de presión, para garantizar una presión mínima en los dispositivos de utilización de $15 \mathrm{~m}$, el agua se envía a la red de riego en el piso $0 \mathrm{y}$ a los sanitarios de todos los apartamentos. En la red de riego ubicada en el piso 0 y en los ramales de distribución de los pisos 1 al 4 fueron necesarias válvulas reductoras de presión para mantener la presión máxima del sistema debajo de $30 \mathrm{~m}$.

\subsection{Costos del sistema hidrosanitario con reutilización de AG}

El costo de inversión del sistema de reutilización de AG se obtuvo como la diferencia del costo del sistema hidrosanitario alternativo ( $i$. e. con reutilización) y el costo del sistema original.

Los costos de operación son representados por la energía necesaria para que el sistema trabaje correctamente y la depreciación de los equipos, que fue calculada suponiendo una vida útil de la bomba de suministro y el sistema de tratamiento de 15 y 10 años respectivamente, de acuerdo con los valores suministrados por los fabricantes de los equipos. Para el cálculo de la depreciación del sistema de tratamiento, se asumió un 80 $\%$ del costo total, para representar las válvulas, aireadores y membranas.

Los costos de mantenimiento se calcularon de acuerdo con los insumos necesarios para el sistema de tratamiento. El salario de un técnico que realiza la limpieza de las membranas estimado en 1.138,7 USD /mes [27]; [28], teniendo en cuenta el tiempo que demanda dicha actividad (i. e. 4 horas cada seis meses) y el costo del vaciado de lodos 29,73 USD /m3), según el tarifario existente de Portugal. No se tuvo en cuenta el costo del encargado del resto del mantenimiento, debido a que estas actividades las puede realizar el personal encargado del mantenimiento de la totalidad del edificio, con previa capacitación.

\subsection{Viabilidad financiera del proyecto}

Para comprobar la viabilidad del proyecto, se realizó un flujo de caja, para determinar el periodo de recuperación de la inversión (PR), el valor presente neto (VPN), la tasa interna de retorno (TIR) y la razón costo/beneficio $(\mathrm{B} / \mathrm{C})$ de acuerdo con las (ecuaciones 5 a 7 ).

$$
\begin{gathered}
V P N=\sum_{i=0}^{n} \frac{F E_{i}}{(1+k)^{i}} \\
0=\sum_{i=0}^{n} \frac{F E_{i}}{(1+T I R)^{i}} \\
B / C=\frac{\sum V P B}{\sum V P C}
\end{gathered}
$$

Donde,

FE, Flujo efectivo;

$\mathrm{k}$, Tasa social de descuento;

i, año del proyecto;

$\mathrm{n}$, vida útil del proyecto;

VPB, valor presente de los beneficios;

VPC, valor presente de los costos.

Para su realización, se tuvo en cuenta la inversión inicial, expresada como egresos iniciales, que representan el costo de implementación del sistema de reutilización. Los ingresos de operación del sistema se representan como el ahorro en el pago de servicios de abastecimiento y saneamiento. Los egresos de operación del sistema están definidos como los costos de la energía consumida por el sistema de reutilización, la depreciación de los equipos y los insumos y la mano de obra necesarios para el mantenimiento del sistema.

El flujo de caja se proyectó suponiendo un horizonte de vida útil de las instalaciones de almacenamiento, tratamiento y distribución de agua de 40 años [29]. Para los costos de los servicios (abastecimiento, alcantarillado y energía eléctrica), de productos para el hogar y equipamientos domésticos y el salario del técnico de mantenimiento, se consideraron tasas anuales de inflación de 3,45 \%, 0,75\% y 3,32\%, respectivamente, correspondiente a la media de la variación del índice de precios del consumidor para cada tipo de consumo y la variación del salario mínimo en Portugal de los últimos 10 años [30]; [31]).

Según Florio [32], la tasa social de descuento para Portugal, calculada por el método de la tasa social de preferencia, es de $4 \%$; no obstante, según Seroa [33], algunos especialistas sugieren el uso de una tasa de 
descuento menor para proyectos donde se verifican beneficios ambientales; por tanto, se sugirió el uso de una tasa social de descuento de $3,5 \%$.

\section{Resultados y discusión}

\subsection{Costos del sistema hidrosanitario con reutilización de AG}

La tabla 9 presenta el presupuesto resumido del sistema hidrosanitario con reutilización de AG (sistema nuevo) y del sistema original; y la tabla 10, los costos de operación y mantenimiento del sistema nuevo.

Los costos en general se ven afectados en gran medida por el sistema de tratamiento (Biorreactores de Membrana, MBR), ya que el mismo presenta un costo del $60 \%$ del total del sistema de reutilización y tiene un alto consumo energético, lo que representa una desventaja importante para considerar la implantación de este tipo de sistema de tratamiento. La instalación de tecnologías alternativas, como reactores de biodiscos (RBC), podría disminuir de forma considerable los costos, de acuerdo con Friedler y Hadari [9], sin afectar la calidad del efluente (Tabla). En proyectos sin restricciones de espacio se pueden aplicar alternativas de tratamiento más económicas, como humedales artificiales, filtros de arena o sedimentadores a continuación de un reactor biológico [13]; [14]; sin embargo, estos tratamientos pueden no proporcionarle al agua un aspecto estético socialmente aceptable [34]. Por tanto, la selección de tecnología para el sistema de reúso de aguas grises es un reto que puede conducir a la eficiencia energética, así como a la obtención de menores costos operativas y de inversión, mayor calidad de las aguas grises y, por ende, mayor aceptación publica del reúso de AG [26].

\subsection{Viabilidad financiera del proyecto}

La tabla 11 presenta un resumen del flujo de caja, donde se observan ganancias a partir del año 23,70 (periodo de retorno). A partir de este flujo, se determinó una TIR de 4,64 \%, un VPN de 14.775,18 USD y una relación B/C de 1.08. De acuerdo con los resultados, se verifica la viabilidad financiera del proyecto, a pesar de los altos costos obtenidos.

Tabla 9. Resumen del presupuesto de construcción

\begin{tabular}{lcc}
\hline Capítulo & Sistema original & Sistema nuevo \\
\hline 1. Redes de drenaje horizontales & USD $1.806,06$ & USD $1.952,40$ \\
2. Instalaciones hidrosanitarias & USD 186.426,05 & USD 205.972,64 \\
2.1. Abastecimiento & USD 142.986,39 & USD 156.533,78 \\
2.2. Drenaje de aguas & USD $43.439,66$ & USD $49.438,87$ \\
3. Equipamiento fijos & USD 118.823,65 & USD $118.823,65$ \\
3.1. Aparatos sanitarios & USD 105.219,40 & USD $105.219,40$ \\
3.2. Cocina & USD 13.604,25 & USD $13.604,25$ \\
4. Sistema de tratamiento de aguas grises & USD & USD $29.893,12$ \\
Costo total & USD 307.055,76 & USD 356.641,82 \\
Diferencia de los sistemas & USD $\mathbf{4 9 . 5 8 6 , 0 6}$ & \\
\hline
\end{tabular}

Fuente: elaboración propia.

Tabla 10. Costos de operación y mantenimiento para el año 2017

\begin{tabular}{llr}
\hline Tipo de egreso & & Costo anual (USD/año) \\
\hline Energía & Sistema de bombeo & USD 192,37 \\
& Sistema de tratamiento & USD 1.110,09 \\
Mantenimiento & Insumos & USD 43,97 \\
& Limpieza de membranas & USD 56,94 \\
Depreciación de los equipos & Vaciado de lodos & USD 148,66 \\
& Sistema de bombeo & USD 620,65 \\
Total & Sistema de tratamiento & USD 2.381,79 \\
\hline
\end{tabular}

Fuente: elaboración propia. 
El periodo de retorno es mayor al de diferentes estudios realizados en diferentes contextos (tabla 12); sin embargo, el valor es cercano al caso de Alemania, donde el sistema de tratamiento elegido y la tarifa de venta de agua son similares al presente caso. La viabilidad financiera de un proyecto depende directamente del número de apartamentos y la tarifa de abastecimiento ( $i$. $e$. mayor número de apartamentos y valor en la tarifa, más factible es financieramente); por tanto, se debe estudiar previamente el contexto de estudio, para elegir el sistema de tratamiento que mejor se ajuste a sus necesidades. Similares resultados son reportados por Oh et al. [26], quienes concluyen que la implementación de sistemas de reúso de aguas grises es factible en términos financieros, especialmente en construcciones con alto consumo de agua (e. $g$. hoteles, restaurantes, establecimientos comerciales, construcciones de oficinas), y, por tanto, un alto potencial de ahorro de agua potable por el reciclaje de AG.

Las limitaciones de la legislación en Portugal en relación con la reutilización de $\mathrm{AG}$ en el sector doméstico estancan el desarrollo comercial de sistemas de tratamiento para este fin. Debe fomentarse investigación aplicada de opciones de tratamiento de AG más rentables e igualmente fiables.

De otro lado, se realizó un análisis de sensibilidad de la tasa de inflación de los servicios, dado que esta inflación es la que origina una mayor variación en los resultados, si se asume el rango de valores de los últimos 10 años $(i$. e. $0,2 \%-8,7 \%$ ). Se encontró que el proyecto solo sería viable para tasas de inflación mayores a 2,87\% (figura 5). Asimismo, se observa una disminución potencial en

Tabla 11. Flujo de caja del proyecto

\begin{tabular}{|c|c|c|c|c|c|c|c|}
\hline \multirow[b]{2}{*}{ Año } & \multicolumn{2}{|c|}{ Ingresos (USD) } & \multicolumn{3}{|c|}{ Egresos (USD) } & \multirow{2}{*}{$\begin{array}{l}\text { Flujo neto } \\
\text { (USD) }\end{array}$} & \multirow{2}{*}{$\begin{array}{c}\text { Flujo neto } \\
\text { acumulado } \\
\text { (USD) }\end{array}$} \\
\hline & Acueducto & Alcantarillado & Energía & Mantenimiento & Depreciación & & \\
\hline 0 & & & & & & $-49.836,90$ & $-49.836,90$ \\
\hline 1 & $4.313,91$ & 780,85 & $1.302,47$ & 249,56 & $3.002,45$ & 540,27 & $-49.296,63$ \\
\hline 10 & $5.853,90$ & $1.059,60$ & $1.767,44$ & 321,86 & $3.211,30$ & $1.612,90$ & $-39.352,65$ \\
\hline 20 & $8.217,70$ & $1.487,46$ & $2.481,13$ & 427,01 & $3.460,44$ & $3.336,59$ & $-14.283,88$ \\
\hline 23 & $9.097,91$ & $1.646,78$ & $2.746,88$ & 464,80 & $3.538,89$ & $3.994,13$ & $-2.975,43$ \\
\hline 24 & $9.411,79$ & $1.703,60$ & $2.841,65$ & 478,13 & $3.565,43$ & $4.230,18$ & $1.254,75$ \\
\hline 30 & $11.536,00$ & $2.088,09$ & $3.483,00$ & 566,51 & $3.728,91$ & $5.845,67$ & $32.116,30$ \\
\hline 40 & $16.194,23$ & $2.931,26$ & $4.889,44$ & 751,58 & $4.018,21$ & $9.466,26$ & $109.403,56$ \\
\hline
\end{tabular}

Fuente: elaboración propia.

Tabla 12. Periodo de retorno en diferentes investigaciones

\begin{tabular}{lcccc}
\hline País/ & $\begin{array}{l}\text { Tarifa } \\
\text { abastecimiento }\end{array}$ & Sistema de tratamiento & $\begin{array}{c}\text { Número de } \\
\text { apartamentos }\end{array}$ & $\begin{array}{c}\text { Periodo de retorno } \\
\text { (años) }\end{array}$ \\
\hline $\begin{array}{l}\text { Este caso de } \\
\text { estudio }\end{array}$ & N.D. & MBR & 72 & 23,7 \\
Siria $^{1}$ & N.D. & $\begin{array}{c}\text { Humedales artificiales } \\
\text { Biofiltro comercial }\end{array}$ & 10 & 7 \\
Colombia $^{2}$ & $0,7 \mathrm{USD} / \mathrm{m}^{3}$ & $\begin{array}{c}\text { Tratamiento químico } \\
\text { (Coagulación/floculación) }\end{array}$ & 10 & 52 \\
Alemania $^{3}$ & $1,9 \mathrm{USD} / \mathrm{m}^{3}$ & RBC & 700 & 11 \\
& & MBR & 70 & 4 \\
Israel $^{3}$ & $1,16 \mathrm{USD} / \mathrm{m}^{3}$ & RBC & 70 & 18 \\
& & MBR & 70 & 6 \\
U.S.A $^{3}$ & $0,51 \mathrm{USD} / \mathrm{m}^{3}$ & RBC & 70 & Inviable \\
Brasil $^{4}$ & N.D. & MBR & 70 & 16 \\
\hline
\end{tabular}

${ }^{1}$ Mourad et al. (2011); ${ }^{2}$ Ardila (2013); ${ }^{3}$ Friedler y Hadari (2006); ${ }^{4}$ Ghisi y Ferreira (2007). N. D.: no dato; RBC: Reactores de Biodisco; MBR: Biorreactor de Membrana 


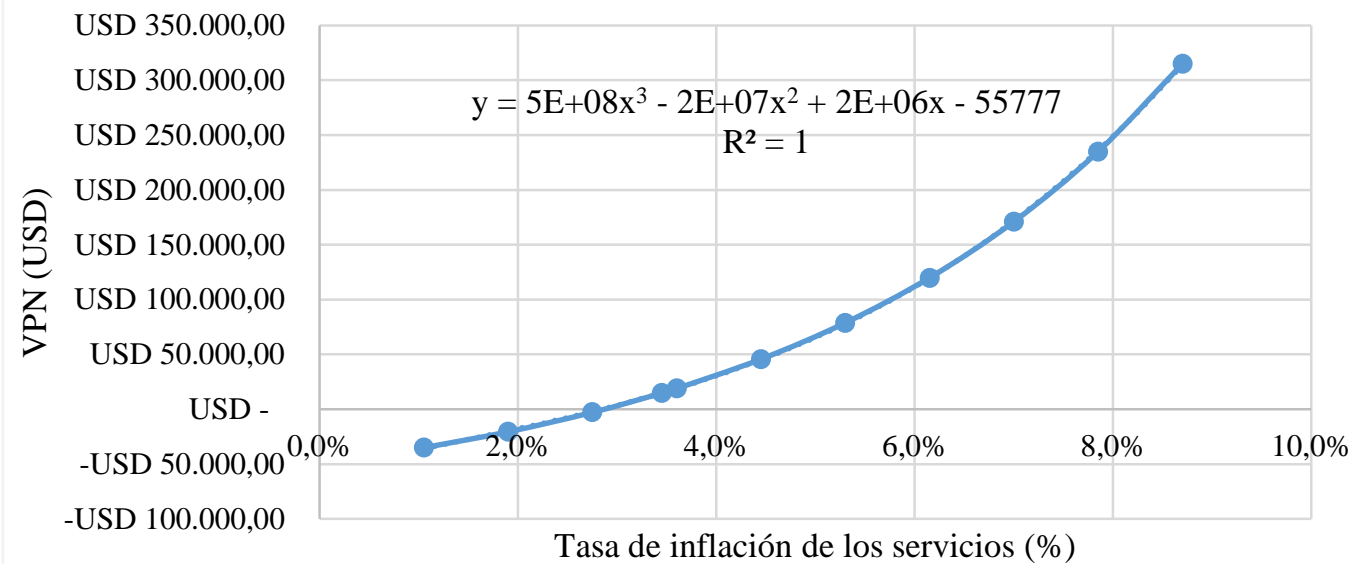

Figura 5. Viabilidad del proyecto en términos de VPN. Fuente: elaboración propia.

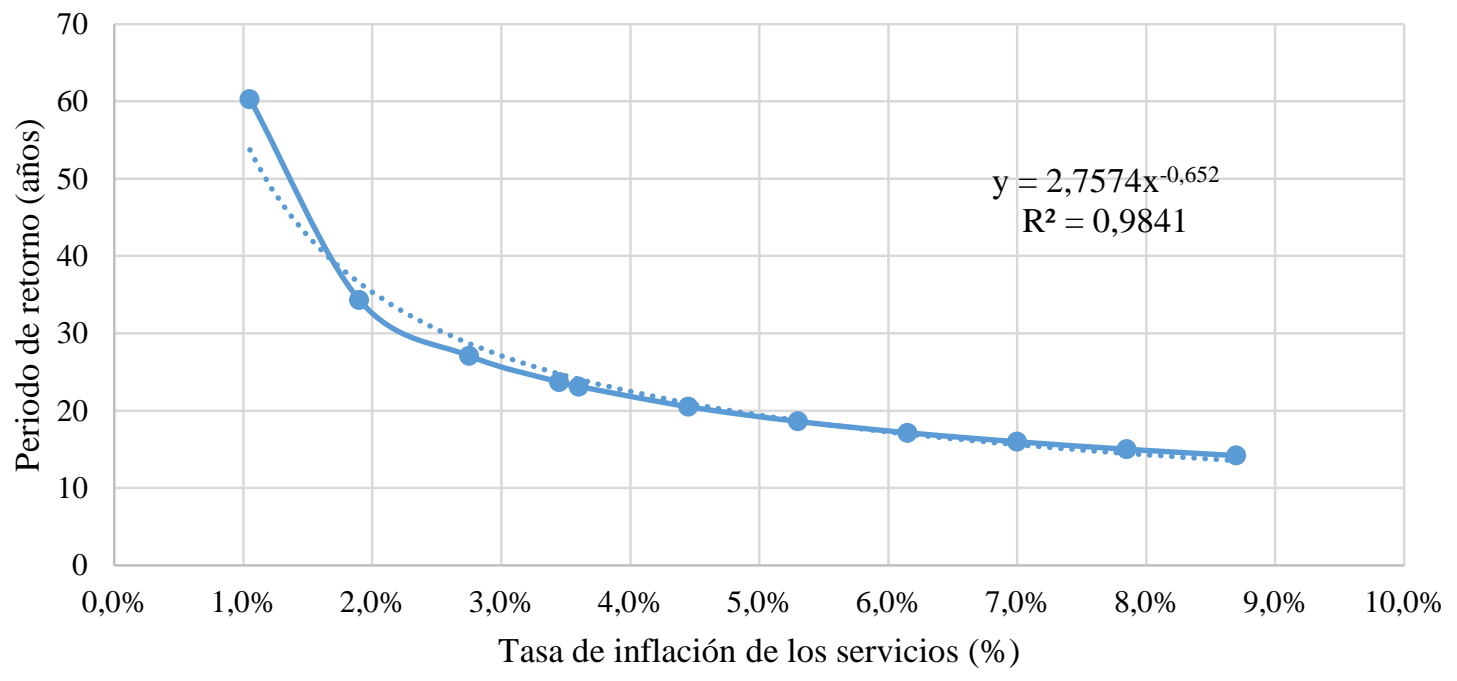

Figura 6. Periodo de retorno según la tasa de inflación de los servicios. Fuente: elaboración propia.

el periodo de retorno, a medida que la tasa de inflación es mayor, y llega hasta 14,19 años para 8,7 \% (figura 6).

Según Gross et al. [20], la implantación de sistemas de reutilización de AG trae beneficios sociales significativos, entre los que están: disminución en el consumo de agua del usuario individual y reducción de los costos del agua, debido a la menor necesidad de búsqueda de nuevas fuentes de agua y la reducción en los costos de producción, tratamiento y transporte del agua. Asimismo, reducción del consumo de agua que reduce la cantidad de AR producidas y, en consecuencia, afecta positivamente el proceso de recolección, transporte y tratamiento de $\mathrm{AR}$, lo que a largo plazo permitiría reducir la capacidad del sistema de tratamiento centralizado, y genera ahorros al público.
Por consiguiente, las autoridades locales o nacionales que busquen mejorar la gestión de los recursos hídricos y traer los beneficios anteriormente explicados a la comunidad general, pueden implementar medidas tales como subsidios por unidad de volumen $\left(\mathrm{m}^{3}\right)$ de agua reutilizada o financiamiento para la implementación de estos sistemas con intereses reducidos [9], [26], lo que favorece la viabilidad financiera del proyecto. Esto teniendo en cuenta los escenarios de escasez hídrica que se visualizan en diferentes contextos urbanos [35].

De otro lado, a pesar de la alta aceptación social del reúso de AG identificada en este estudio (i. e. $80 \%$ ), es necesario el desarrollo de proyectos piloto en Portugal que muestren las tecnologías empleadas para el tratamiento de las AG, el sistema de reúso del AG en el 
ámbito de las unidades residenciales, los riesgos reales a los usuarios, los beneficios financieros de la implementación del sistema. Esto podría contribuir a incrementar la aceptación de este tipo de alternativas en el contexto de Portugal.

\section{Conclusiones}

De acuerdo con los resultados obtenidos en el presente artículo, se concluye lo siguiente:

- La implementación de un sistema de reutilización de AG en el caso de estudio es financieramente viable (i. e. periodo de retorno de 23,70 años, TIR de 4,64 \%, VPN de 14.775,18 USD y relación B/C de $1,08)$, siempre que la tasa de inflación de los servicios se mantenga por encima de $2,87 \%$; esto es posible teniendo en cuenta la escasez de recursos hídricos y energéticos al que se enfrenta el mundo, lo cual generaría un sobrecosto en dichos servicios. Además, es necesario cuantificar los beneficios y posibles costos ambientales y sociales para demostrar la viabilidad económica y social del proyecto.

Los indicadores financieros podrían ser más atractivos con la implementación de sistemas de instalación de estos sistemas, asistencia técnica para el diseño y operación y mantenimiento de los sistemas de reúso de aguas grises.

\section{Agradecimientos}

Los autores agradecen a la Universidad Industrial de Santander y a la Universidad de Minho, por el soporte para la elaboración de este manuscrito.

\section{Referencias}

[1] "Informe de las Naciones Unidas sobre los recursos hídricos en el mundo 2015. Agua para un mundo sostenible: datos y cifras", UNESCO, División de Ciencias del Agua, Colombella, 2015.

[2] "Informe Mundial de las Naciones Unidas sobre el Desarrollo de los Recursos Hídricos 2017. Aguas residuales: El recurso desaprovechado", WWAP Programa Mundial de Evaluación de los Recursos Hídricos de las Naciones Unidas, París, 2017.

[3] “Implementación 2012- 2020”, PNUEA Programa Nacional para o uso eficiente da agua, Agencia Portuguesa do Ambiente, I.P., 2012.

[4] APDA. Água, quem é quem. Suplementaria. Lisboa, Portugal, 1999.
[5] H. Marecos do Monte, y A. Albuquerque,. Guia técnico 14. Reutilização de aguas residuais. Lisboa: Entidade Reguladora dos Serviços de Águas e Resíduos, Instituto Superior de Engenharia de Lisboa, 2013.

[6] T. Asano, A. D.Levine, "Wastewater reclamation, recycling and reuse: past, present, and future," Water Sci. Technol., vol. 33, no. 10-11, 1996. doi: 10.1016/02731223(96)00401-5

[7] E. R. Oviedo-Ocaña, I. Dominguez, S. Ward, M. L. Rivera-Sanchez, y J. M. Zaraza-Peña, "Financial feasibility of end-user designed rainwater harvesting and greywater reuse systems for high water use households," Environ. Sci. Pollut. Res., vol. 25, no. 20, pp. 1920019216, 2018. doi: 10.1007/s11356-017-8710-5

[8] C. Matos, "Reutilização de água. Utilização de águas cinzentas in situ", tesis doctoral, Universidade de Trásos-Montes e Alto Douro, Vila Real, Portugal, 2010.

[9] E. Friedler y M. Hadari, "Economic feasibility of onsite greywater reuse in multi-storey buildings," Desalination, vol. 190, no. 1-3, pp. 221-234, 2006. doi: 10.1016/j.desal.2005.10.007

[10] N. Atanasova, M. Dalmau, J. Comas, M. Poch, I. Rodriguez-Roda, y G. Buttiglieri, "Optimized MBR for greywater reuse systems in hotel facilities," J. Environ. Manage., vol. 193, pp. 503-511, 2017. doi: 10.1016/j.jenvman.2017.02.041

[11] J. March, M. Gual, y F. Orozco, "Experiences on greywater re-use for toilet flushing in a hotel (Mallorca Island, Spain)," Desalination, vol. 164, no. 3, pp. 241247, 2004. doi: 10.1016/S0011-9164(04)00192-4

[12] M. Ardila, "Viabilidad técnica y económica del aprovechamiento de aguas grises domésticas", trabajo de fin de maestría, Universidad Nacional de Colombia, Colombia, 2013.

[13] K. A. Mourad, J. C. Berndtsson, y R. Berndtsson, "Potential fresh water saving using greywater in toilet flushing in Syria," J. Environ. Manage., vol. 92, no. 10, pp. 2447-2453, 2011. doi: 10.1016/j.jenvman.2011.05.004

[14] E. Ghisi y D. F. Ferreira, "Potential for potable water savings by using rainwater and greywater in a multistorey residential building in southern Brazil," Build. Environ., vol. 42, no. 7, pp. 2512-2522, 2007. doi: 10.1016/j.buildenv.2006.07.019 
[15] S. Godfrey, P. Labhasetwar, y S. Wate, "Greywater reuse in residential schools in Madhya Pradesh, IndiaA case study of cost-benefit analysis," Resour. Conserv. Recycl., vol. 53, no. 5, pp. 287-293, 2009. doi: 10.1016/j.resconrec.2009.01.001

[16] I. Domínguez, S. Ward, J. Mendoza, C. Rincón, y E. Oviedo-Ocaña, "End-User Cost-Benefit Prioritization for Selecting Rainwater Harvesting and Greywater Reuse in Social Housing", Water, vol. 9, no. 7, 2017. doi: 10.3390/w9070516

[17] M. Almeida-Vieira y P. Vieira e Rita Ribeiro, Uso eficiente da água no sector urbano. Lisboa: Instituto Regulador de Águas e Resíduos, 2006

[18] J. Melo-Baptista, M.C. Almeida, P. Vieira, A. M. Silva, R. Ribeiro, R. M. Fernando, A. Serafim, I. Alves, M. R Cameira, "Uso Eficiente da Água - Proposta de programa nacional", Relatório 286/01-NES Laboratório Nacional de Engenharia Civil, Lisboa, Agosto de 2001.

[19] A. Prathapar, A. Jamrah, M. Ahmed, S. Al Adawi, S. Al Sidairi, y A. Al Harassi, "Overcoming constraints in treated greywater reuse in Oman", Desalination, vol. 186, no. 1-3, pp. 177-186, 2005. doi: 10.1016/j.desal.2005.01.018

[20] A. Gross, A. Maimon, Y. Alfiya, E. Friedler. Greywater Reuse. Boca Raton, Florida: CRC Press, 2015.

[21] C. Gonçalves, "Viabilidade da reutilização de águas residuais em habitações unifamiliares. Dissertação de Mestrado", trabajo de fin de maestría, Universidade do Minho, Portugal, 2011.

[22] M. Pidou, F. A. Memon, T. Stephenson, B. Jefferson, y P. Jeffrey, "Greywater recycling: treatment options and applications," Proc. Inst. Civ. Eng. - Eng. Sustain., vol. 160, no. 3, pp. 119-131, 2007. doi: 10.1680/ensu.2007.160.3.119

[23] P. Vieira, A. Moura, J. Melo-Baptista, M. Do Céu Almeida, R. Ribeiro, "Inquérito aos hábitos de utilização e consumos de agua na habitação," in $10^{\circ}$ Encontro Nacional de Saneamento Básicol $10^{\circ}$ Simpósio LusoBrasileiro de Engenharia Sanitária e Ambienta, Braga, Portugal, 2002.

[24] "EcoFamílias- Água. Relatório final dezembro 2008- janeiro 2010", Associação Nacional de Conservação da Natureza \& Veolia Água, Março 2010.
[25] "PNUEA Programa Nacional para o uso eficiente da água, Versão preliminar", Ministerio do ambiente e ordenamento do territorio, Outubro 2001.

[26] K. S. Oh, J. Y. C. Leong, P. E. Poh, M. N. Chong, y E. Von Lau, "A review of greywater recycling related issues: Challenges and future prospects in Malaysia," $J$. Clean. Prod., vol. 171, pp. 17-29, 2018. doi: 10.1016/j.jclepro.2017.09.267

[27] "Enquadramento das profissões e categorias profissionais em níveis de retribuição", Boletin do Trabalho e Emprego, no. 26, 2018.

[28] Compare seu salário, Compare seu salário com pessoas na mesma ocupação em três passos, 2017. [En línea]. Disponible en: https://meusalario.pt/inicio/salario/compare-seusalario?job-id=3112010000000\#/.

[29] J. Jiménez, Manual para el diseño de sistemas de agua potable y alcantarillado sanitario. Mexico: Universidad Veracruzana, 2010.

[30] Taxa de Inflação (Taxa de Variação do Índice de Preços no Consumidor): total e por consumo individual por objetivo - Portugal, 2017. [en línea]. Disponible en: http://www.pordata.pt/Portugal/Taxa+de+Infla\%C3\%A $7 \% \mathrm{C} 3 \% \mathrm{~A} 3 \mathrm{o}+(\mathrm{Taxa}+\mathrm{de}+$ Varia $\% \mathrm{C} 3 \% \mathrm{~A} 7 \% \mathrm{C} 3 \% \mathrm{~A} 3 \mathrm{O}+\mathrm{do}$ $+\% \mathrm{C} 3 \% 8$ Dndice+de+Pre\% $3 \% \mathrm{~A} 7 \mathrm{os}+$ no+Consumidor) +total+e+por+consumo+individual+por+objectivo2315.

[31] Portugal, S. SMI en Portugal 2017, 2017. [en línea] Disponible http://www.datosmacro.com/smi/portugal.

[32] M. Florio, "Cost-benefit analysis and the European union cohesion fund: On the social cost of capital and labour," Reg. Stud., vol. 40, no. 2, pp. 211-224, 2006. doi: 10.1080/00343400600600579

[33] R. Seroa, Economía ambiental. Brasil: Editora FGV, 2006.

[34] M. L. Griffioen, y S.Natha, "Improving a greywater reuse unit for the purpose of toilet flushing in a student residence at the University of Johannesburg, South Africa," In World Environmental and Water Resources Congress 2013: Showcasing the Future, Cincinnati, Ohio, pp. 3090-3096.

[35] A. Castillo-Rodríguez, M. Castro-Chaparro, A. Gutiérrez-Malaxechebarría, C. Aldana-Gaviria, "Estimación sectorial de la huella hídrica de la ciudad de 
Bogotá generada en el año 2014," Rev. UIS Ing., vol. 17, no. 2, pp. 19-32, 2018. doi: 10.18273/revuin.v17n22018002 